\title{
Colonic Metastases from a Luminal A Type Invasive Ductal Carcinoma Breast: A Rare Case Report
}

\begin{abstract}
Metastatic carcinoma breast involving hepatobiliary system and peritoneum is a common and well-described entity. However, colonic luminal involvement by breast metastases is rare and its early recognition is important for accurate management planning. We report a case of an elderly woman with luminal A type invasive ductal carcinoma (IDC) breast, initially controlled/stabilized on hormonal treatment and later progressed to involve colon presenting with intestinal obstructive symptoms. Lobular carcinoma breast which constitutes around $10 \%$ of breast cancers is more likely to have gastrointestinal tract (GIT) involvement. On the other hand, GIT metastasis from IDC breast is quite rare. Given the progressively increasing survival with current and forthcoming novel treatment modalities, it is very important to recognize and understand unusual presentation of metastatic breast cancer, such as colonic metastasis, for timely treatment planning and implementation.
\end{abstract}

Keywords: Carcinoma breast, colonic metastases, invasive ductal carcinoma, unusual metastasis

\section{Introduction}

Breast cancer is the most common cancer among females, worldwide. ${ }^{[1]}$ Various prognostic factors in a case of breast cancer include molecular phenotype, hormonal and human epidermal growth factor receptor 2 (HER2) status, size, grade of tumor, stage of disease, and site of metastasis. ${ }^{[2]}$

Common secondaries in a case of breast primary include, but are not limited to, nodes, bones, lungs, liver, brain, etc. Involvement of gastrointestinal tract (GIT) is uncommon, and still rarer is the colonic lumen involvement by metastasis.

We report an unusual case of luminal A type of metastatic invasive ductal carcinoma (IDC) breast, who had brain metastasis at the time of presentation and diagnosis. She was initially stable on hormonal treatment and survived unusually longer than expected with brain metastasis ${ }^{[3,4]}$ and then progressed and presented with intestinal obstructive symptoms and bleeding per rectum. On investigations, she was eventually diagnosed to have colonic metastases.

\section{Case Report}

A 73-year-old postmenopausal woman with comorbidities of hypertension, type 2

This is an open access journal, and articles are distributed under the terms of the Creative Commons Attribution-NonCommercial-ShareAlike 4.0 License, which allows others to remix, tweak, and build upon the work non-commercially, as long as appropriate credit is given and the new creations are licensed under the identical terms.

For reprints contact: WKHLRPMedknow_reprints@wolterskluwer.com diabetes, sarcoidosis, and diabetic nephropathy was diagnosed to have carcinoma breast in January 2014. Biopsy and histopathological examination from breast lesion revealed IDC, positive for estrogen receptor (ER) and progesterone receptor (PR), with Allred score of $6 / 8$ and 5/8, respectively. HER2 was negative by immunohistochemistry (IHC). Positron emission tomography-computed tomography (PET-CT) revealed heterogeneously enhancing lobulated soft-tissue attenuation involving all four quadrants of the right breast and overlying skin involvement, as well as extensive skeletal, nodal, peritoneal, mesenteric, and brain metastases. The patient was not willing for any radiotherapy or chemotherapeutic intervention. Initially, she had taken alternative therapy in the form of Ayurvedic medicines for 6 months. We started her on tablet exemestane $25 \mathrm{mg}$ once a day (OD) in July 2014. Initially, her symptoms improved followed by disease stabilization. She was apparently all right for a year on hormonal treatment till June 2015, when she developed respiratory failure and uncontrolled diabetes which were managed. PET-CT was done for disease evaluation which showed overall progressive disease (PD). There was marked interval decrease in size, fludeoxyglucose (FDG) avidity of breast lesion, and complete

How to cite this article: Sehrawat A, Kotwal SA, Gupta D. Colonic metastases from a luminal A type invasive ductal carcinoma breast: A rare case report. Indian J Med Paediatr Oncol 2020;41:96-8.

\section{Amit Sehrawat ${ }^{1}$, Sumedha Ahal Kotwal', Deni Gupta ${ }^{3}$}

${ }^{1}$ Department of Medical Oncology Haematology, All India Institute of Medical Sciences, Rishikesh, Uttarakhand, India, ${ }^{2}$ Department of Pathology, Dharamshila Narayana Superspeciality Hospital, New Delhi, India, ${ }^{3}$ Department of Medical Oncology, Dharamshila Narayana Superspeciality Hospital, New Delhi, India

Submitted: 09-May-2018 Accepted in Revised Form: 21-Jun-2018

Published: 24-Apr-2020

Address for correspondence:

Dr. Amit Sehrawat

Assistant Professor Medical Oncology Haematology, All India Institute of Medical

Sciences, Rishikesh, Uttarakhand, India.

E-mail: dramitsehrawat@gmail. com

Access this article online

Website: www.ijmpo.org

DOI: 10.4103/ijmpo.ijmpo_109_18

Quick Response Code:

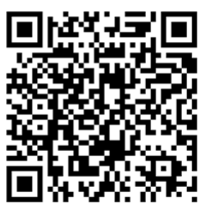


metabolic resolution of axillary lymphadenopathy. However, FDG avidity of the pulmonary nodules increased with increased interval in FDG avidity and decreased size of skeletal lesions. Increased FDG avidity and size of adrenal, omental, mesenteric, and peritoneal deposits was also seen. She had not any gastrointestinal symptoms or any evidence of PET avid disease in the colon. Despite her symptoms, the patient refused cytotoxic chemotherapy. She was switched to tablet letrozole $2.5 \mathrm{mg}$ OD along with injection denosumab $120 \mathrm{mg}$, every 4 weekly. She remained clinically stable over the next 6 months till January 2016, when she developed acute respiratory symptoms in the form of cough and progressive dyspnea. On PET-CT, there was evidence of PD (increased size and FDG avidity of primary breast lesion and metastatic lesions). After clinical stabilization, she was put on fulvestrant $500 \mathrm{mg}$ every 4 weekly and tablet anastrozole $1 \mathrm{mg}$ OD. Four months later, she developed seizures with progressive decline in cognitive functions along with progressive constipation. Magnetic resonance imaging brain done in April 2016 showed multiple nodular mass lesions in cerebral and cerebellar and frontoparietal region with perilesional edema, suggestive of brain metastasis. PET-CT was consistent with $\mathrm{PD}$, with metabolically active mural lesion involving proximal transverse colon, distal end of ileum, adjacent to ileocecal junction [Figure 1]. Her carcinoembryonic antigen levels were $173.69 \mathrm{ng} / \mathrm{ml}$. Colonoscopy revealed a stricture with luminal narrowing in the transverse colon along with an ulceroproliferative growth in the proximal ascending colon [Figure 2]. Histopathology of the colon lesion biopsy revealed moderately differentiated adenocarcinoma in the mucosa with cellular infiltrative pattern, and IHC showed CK, CK7, CEA, P-120, GCDFP positivity and CK-20, CDX-2 negativity, suggestive of metastatic adenocarcinoma from the breast primary [Figure 3]. Symptomatic treatment was given as surgical intervention was refused. She was managed with best supportive care and eventually succumbed to her disease.

\section{Discussion}

Breast cancer survival and overall prognosis, even in metastatic setting, have evolved significantly over the past few years. With availability of new therapeutic modalities and novel chemotherapeutic and targeted agents coupled with better understanding of pathology of breast cancer, patient survival is prolonged. Therefore, recognition of unusual pattern-like GIT secondaries becomes important for their early diagnosis as well as timely appropriate intervention or change of treatment strategy.

This case is rare in terms of unusually prolonged survival, ${ }^{[3,4]}$ in spite of brain metastasis at presentation without any radiotherapeutic or chemotherapy intervention. The case also highlights the role targeting of hormone receptors and its impact on survival, even in patient with extensive metastatic disease. Although lymph nodes, lung,

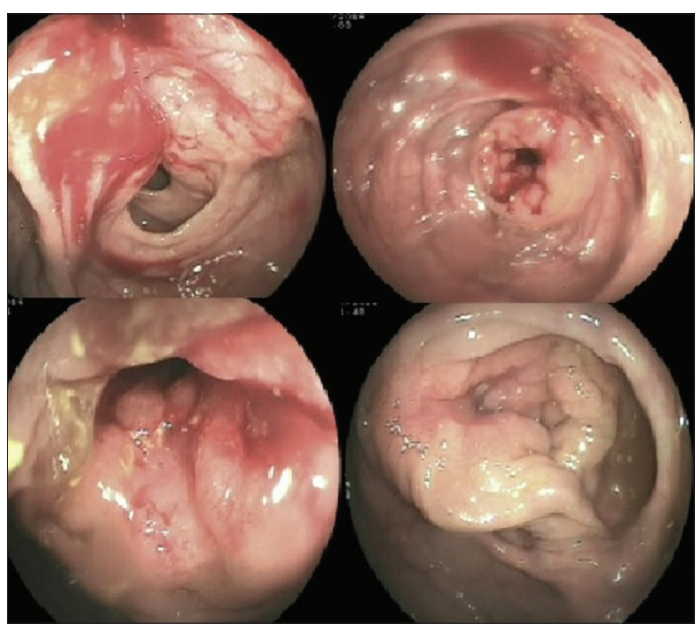

Figure 1: Colonoscopy showing ulceroproliferative lesion with obstruction in ascending colon and transverse colon

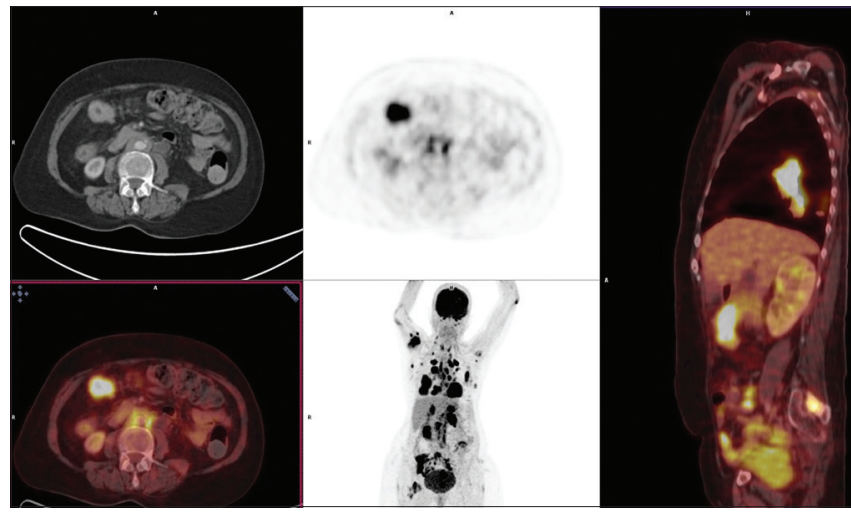

Figure 2: ${ }^{18}$ Fludeoxyglucose-positron emission tomography-computed tomographic images depicting metastatic disease burden from primary right breast lesion, including transverse colon and ileocecal junction

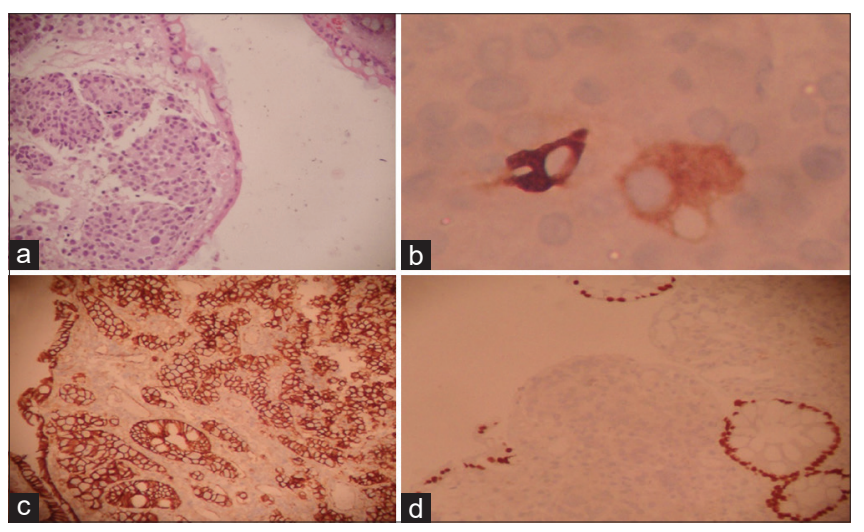

Figure 3: Photomicrographs showing histology and immunohistochemistry profile of colonic deposits. (a) Tumor deposits in colonic mucosa beneath surface epithelium ( $H$ and $E, \times 100$ ). (b) Cytoplasmic GCDFP-15 expression by few tumor cells $(\mathrm{IHC}, \times 400)$. (c) Membranous $\mathrm{p} 120$ positivity in tumor cells and colonic epithelium as internal control $(\mathrm{IHC}, \times 200)$. (d) Tumor cells nonimmunoreactive to CDX2 with positive normal epithelium as internal control $(\mathrm{IHC}, \times 200)$

liver, or bones are the most common sites for distant metastases, a few cases of GIT involvement have been reported. As metastases to GIT from breast primary are very 
rarely recognized clinically, its true incidence is not known. Our case is one of the very few reported cases of colonic metastases from breast primary, worldwide. ${ }^{[5]}$ This elderly woman had de novo metastatic breast cancer (MBC) with brain metastasis at the time of presentation, which as such is a poor prognostic marker independent of other risks. ${ }^{[2]}$

Invasive lobular carcinoma breast is known to metastasize to GIT, but reports of IDC involving GIT are scarce. ${ }^{[6,7]}$ GIT metastases from breast have been reported to stomach, small intestine, and colorectal region. The stomach is the most common site of gastrointestinal involvement by $\mathrm{MBC}$, with an incidence of up to $15 \%$ at autopsy. ${ }^{[8]}$ Common pattern observed in GIT involvement was infiltrative pattern as in our case. Colonic involvement is common with some autopsy series showing colonic metastases in up to $12 \%$ of patients with breast cancer. ${ }^{[9]}$ A case series by Nazareno et al. reported that autopsy involvement of the colon is much higher than actual clinical presentation of colonic metastasis. ${ }^{[10]}$ Carcinoma of the breast is the most frequent source of hematogenous metastases to the large bowel, but a peritoneal or lymphatic route is also possible. ${ }^{[11]}$

The median time to the development of metastases is about 4 or 5 years. Patients can present with obstructive symptoms and/or bleeding per rectum such as a primary in colon or may mimic irritable bowel disease, Crohn's disease, or ulcerative colitis. ${ }^{[12]}$

Classically described radiological finding of intestinal primary, apple-core lesion, is uncommon. Endoscopy and biopsy from the growth are the only diagnostic modalities to accurately differentiate colonic primary from secondary breast cancer lesions. The use of appropriate IHC markers is very essential in such situations, as in our patient. Positivity for ER, PR, CK7, and GCDFP15, as well as negativity for CA19-9, CK20, and CDX2, suggests a breast origin of a colonic lesion, especially in a known case of breast cancer. ${ }^{[13]}$ Ductal breast neoplasms have membranous staining, whereas lobular cancers show diffuse cytoplasmic expression of P120 catenin. ${ }^{[14]}$ This can also help in differentiating types of secondary deposits as seen in the present case, where deposits were seen in the mucosa.

Thus, GIT metastasis from breast carcinoma may mimic primary bowel neoplasm in clinical presentation, and IHC aids in differentiating between the two.

\section{Conclusion}

Given the progressively increasing incidence and survival of carcinoma breast patients with currently available and evolving new modalities, we have to expect and recognize unusual presentations of $\mathrm{MBC}$, such as colonic metastasis, for timely intervention planning and implementation.

\section{Declaration of patient consent}

The authors certify that they have obtained all appropriate patient consent forms. In the form the patient(s) has/have given his/her/their consent for his/her/their images and other clinical information to be reported in the journal. The patients understand that their names and initials will not be published and due efforts will be made to conceal their identity, but anonymity cannot be guaranteed.

\section{Financial support and sponsorship}

Nil.

\section{Conflicts of interest}

There are no conflicts of interest.

\section{References}

1. Ferlay J, Soerjomataram I, Dikshit R, Eser S, Mathers C, Rebelo $\mathrm{M}$, et al. Cancer incidence and mortality worldwide: Sources, methods and major patterns in GLOBOCAN 2012. Int $\mathrm{J}$ Cancer 2015;136:E359-86.

2. Largillier R, Ferrero JM, Doyen J, Barriere J, Namer M, Mari V, et al. Prognostic factors in 1,038 women with metastatic breast cancer. Ann Oncol 2008;19:2012-9.

3. Leone JP, Lee AV, Brufsky AM. Prognostic factors and survival of patients with brain metastasis from breast cancer who underwent craniotomy. Cancer Med 2015;4:989-94.

4. Brogi E, Murphy CG, Johnson ML, Conlin AK, Hsu M, Patil S, et al. Breast carcinoma with brain metastases: Clinical analysis and immunoprofile on tissue microarrays. Ann Oncol 2011;22:2597-603.

5. Uygun K, Kocak Z, Altaner S, Cicin I, Tokatli F, Uzal C. Colonic metastasis from carcinoma of the breast that mimics a primary intestinal cancer. Yonsei Med J 2006;47:578-82.

6. McLemore EC, Pockaj BA, Reynolds C, Gray RJ, Hernandez JL, Grant CS, et al. Breast cancer: Presentation and intervention in women with gastrointestinal metastasis and carcinomatosis. Ann Surg Oncol 2005;12:886-94.

7. Critchley AC, Harvey J, Carr M, Iwuchukwu O. Synchronous gastric and colonic metastases of invasive lobular breast carcinoma: Case report and review of the literature. Ann R Coll Surg Eng1 2011;93:e49-50.

8. Joffe N. Metastatic involvement of the stomach secondary to breast carcinoma. Am J Roentgenol Radium Ther Nucl Med 1975;123:512-21.

9. Cifuentes N, Pickren JW. Metastases from carcinoma of mammary gland: An autopsy study. J Surg Oncol 1979;11:193-205.

10. Nazareno J, Taves D, Preiksaitis HG. Metastatic breast cancer to the gastrointestinal tract: A case series and review of the literature. World J Gastroenterol 2006;12:6219-24.

11. Fujimoto D, Hirono Y, Goi T, Yamaguchi A. Sigmoid colonic metastasis by lymphatic spread occurring with unilateral krukenberg tumor considered to be caused by stage IA early gastric cancer: A case report. Oncol Lett 2016;11:668-72.

12. Villa Guzmán JC, Espinosa J, Cervera R, Delgado M, Patón R, Cordero García JM. Gastric and colon metastasis from breast cancer: Case report, review of the literature, and possible underlying mechanisms. Breast Cancer (Dove Med Press) 2017;9:1-7.

13. Birla R, Mahawar KK, Orizu M, Siddiqui MS, Batra A. Caecal metastasis from breast cancer presenting as intestinal obstruction. World J Surg Oncol 2008;6:47.

14. Li X, Schwartz MR, Ro J, Hamilton CR, Ayala AG, Truong LD, et al. Diagnostic utility of E-cadherin and P120 catenin cocktail immunostain in distinguishing DCIS from LCIS. Int J Clin Exp Pathol 2014;7:2551-7. 